\title{
Modification of refractive index by a single femtosecond pulse confined inside a bulk of a photorefractive crystal
}

\author{
Eugene G. Gamaly* \\ Laser Physics Centre, Research School of Physics and Engineering, The Australian National University, Canberra, \\ Australian Capital Territory 0200, Australia
}

Saulius Juodkazis

Centre for Micro-Photonics, Faculty of Engineering and Industrial Sciences Swinburne, University of Technology, Hawthorn, Victoria 3122, Australia

and Research Institute for Electronic Science, Hokkaido University, North 21-West 10, CRIS Building, Kita-ku, Sapporo 001-0021, Japan

Vygantas Mizeikis

Division of Global Research Leaders and Research Institute for Electronics, Shizuoka University, 3-5-1 Johoku, Naka-ku, Hamamatsu 432-8561, Japan

Hiroaki Misawa

Research Institute for Electronic Science, Hokkaido University, North 21-West 10, CRIS Building, Kita-ku, Sapporo 001-0021, Japan

Andrei V. Rode and Wieslaw Krolikowski

Laser Physics Centre, Research School of Physics and Engineering, The Australian National University, Canberra, Australian Capital Territory 0200, Australia

(Received 5 May 2009; revised manuscript received 11 January 2010; published 23 February 2010)

\begin{abstract}
We demonstrate that the interaction of intense femtosecond pulse with photorefractive crystal at conditions close to the optical-breakdown threshold differs drastically from that of long pulse and cw illumination. Our theoretical estimations show that the high number density of excited electrons modifies the dielectric function leading to the transient negative change in the refractive index, $\Delta n / n_{0} \sim-10^{-2}$ that vanishes on nanosecond time scale. Moreover, the high-frequency laser field, two orders of magnitude larger than the field of spontaneous polarization, prevents the stationary charge distribution during the pulse. The diffusion and recombination of charge carriers continues over a nanosecond time scale, after the end of the pulse. The main driving force for the current after the pulse is the field of spontaneous polarization in the ferroelectric medium: the current terminates when the field of charge separation balances this field. We show here that the stationary modification of refractive index according to this model is then independent of the polarization of the pump light beam, in agreement with experiments, and saturates at $\Delta n \approx 10^{-3}$ in semiquantitative fit to the experimental data.
\end{abstract}

DOI: 10.1103/PhysRevB.81.054113

PACS number(s): 81.16.-c, 42.70.Ln, 73.50.Pz

\section{INTRODUCTION}

The interactions of long laser pulses and continuous wave (cw) lasers with the photorefractive materials were studied thoroughly during the past decades and the mechanisms for refractive-index modification at low intensities are well understood. ${ }^{1-5}$ Recently the fs-laser photorefractive interactions have been found attractive for applications in threedimensional (3D) optical memories, ${ }^{6-8}$ photonic crystals fabrication, ${ }^{9}$ waveguide formation, ${ }^{10,11}$ second-harmonic generation, ${ }^{12}$ parametric light conversion, ${ }^{13}$ and terahertz ${ }^{14,15}$ applications. Femtosecond laser writing allows for very fast processing and design of the read-write-erase memory devices. However, the studies revealed several distinctive features distinguishing interaction of short pulses from those with long and cw lasers. Below we consider in detail the interaction of powerful femtosecond pulses with these crystals and compare it with the low-intensity long pulse interactions.

The major difference between femtosecond and long pulse interaction with photorefractive crystals relates to the fact that in the former case the laser field of high intensity acts over the period much shorter than the major relaxation times. Indeed, it follows from the experiments with lithium niobate $^{16,17}$ that the lowest intensity at which the change in the refraction index produced by single $800 \mathrm{~nm}, 150 \mathrm{fs}$ pulses ( $6 \mathrm{~nJ}$ per pulse) could still be detected is on the order

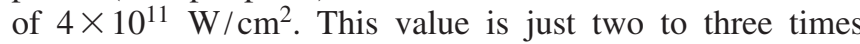
lower than the ionization threshold for the dielectrics. ${ }^{18,19}$ Therefore the number density of excited electrons in the conduction band, as we demonstrate below, is two orders of magnitude higher than that produced by long pulse and $\mathrm{cw}$ lasers. At such intensity the electrons are excited from all constituent atoms in contrast to conventional photovoltaic effect where only dopants (iron and other transition metals) are excited. Hence the number density of all excited electrons is almost two orders of magnitude larger than the number density of doped atoms.

Before proceeding further let us recall the main processes contributing to formation of a diffractive structure in the photorefractive materials under light illumination at low 
intensity. ${ }^{20}$ The photoexcitation of free carriers into the conduction band occurs during the short interaction (pulse) time. Their subsequent transport is a result of the combined action of electric fields: a local field of spontaneous polarization, and diffusion field due to gradients of charge density and temperature. The recombination goes simultaneously while ions remain fixed. Because the recombination time is longer than the pulse duration the carriers are transported by the current and recombine after the end of the pulse in a locations different from where they were created. The current stops when the gradients in spatial distribution of charge carriers are created and corresponding electric field of charge separation balances the field of spontaneous polarization. The field of charge separation then induces a refractive-index modulation via the electro-optic effect similarly to that in a long pulse or cw case. For the memory applications it is also important to define the time for the transition to quasisteady charge distribution and total lifetime of the charge distribution that defines the reliability of this process. In this work we describe, step by step, all the processes constituting the above scenario.

The structure of the paper is as follows. First, (Sec. II) we deduce from the experiments the range of fs-laser parameters for the reversible formation of diffractive changes and recollect the properties of lithium niobate. In Sec. III the known features of the low-intensity regime are briefly recollected followed by the thorough description of the high-intensity regimes, both regimes are compared. Then, the theoretical results are compared to the available experimental data. Finally, Sec. IV contains summary of our findings and conclusions.

\section{EXPERIMENTAL CONDITIONS}

\section{A. Laser pulse parameters}

We consider formation of microvolumes of photomodified refractive index by a single laser pulse at tight focusing conditions $^{8,19}$ as shown in Fig. 1(a). Typical femtosecond laser-exposure conditions were: a 150 fs pulse with the peak intensity $0.5-1.5 \mathrm{TW} / \mathrm{cm}^{2}$ at a $800 \mathrm{~nm}$ wavelength with energy per pulse 8-24 $\mathrm{nJ}$ and focal spot area of $\sim 10^{-7} \mathrm{~cm}^{2}$. For simplicity, we consider here a spherical aberration-free focusing inside photorefractive materials. The actual lightintensity distribution is more complex as illustrated in the inset of Fig. 1(a) by 3D finite difference time domain (FDTD) simulations (Lumerical). However, changes in the focal spot location and axial elongation can be, in principle, compensated for by the appropriate phase front shaping. ${ }^{22}$

The lowest light intensity at which the change in the refractive index could still be recorded (and measured experimentally via light diffraction on optically written index grating ${ }^{8}$ ) was $3.8 \pm 0.5 \mathrm{~nJ}$ in $\mathrm{LiNbO}_{3}: \mathrm{Fe}$ and $5.2 \pm 0.5 \mathrm{~nJ}$ in undoped $\mathrm{LiNbO}_{3}$, respectively, for focusing by an objective lens with numerical aperture $N A=0.55$.

\section{B. Properties of lithium niobate}

Single crystals (Y cut) of near stoichiometric $(\mathrm{Li} / \mathrm{Nb}=49.85 / 50.15)$ undoped and $400 \mathrm{ppm}$ Fe doped were (a)
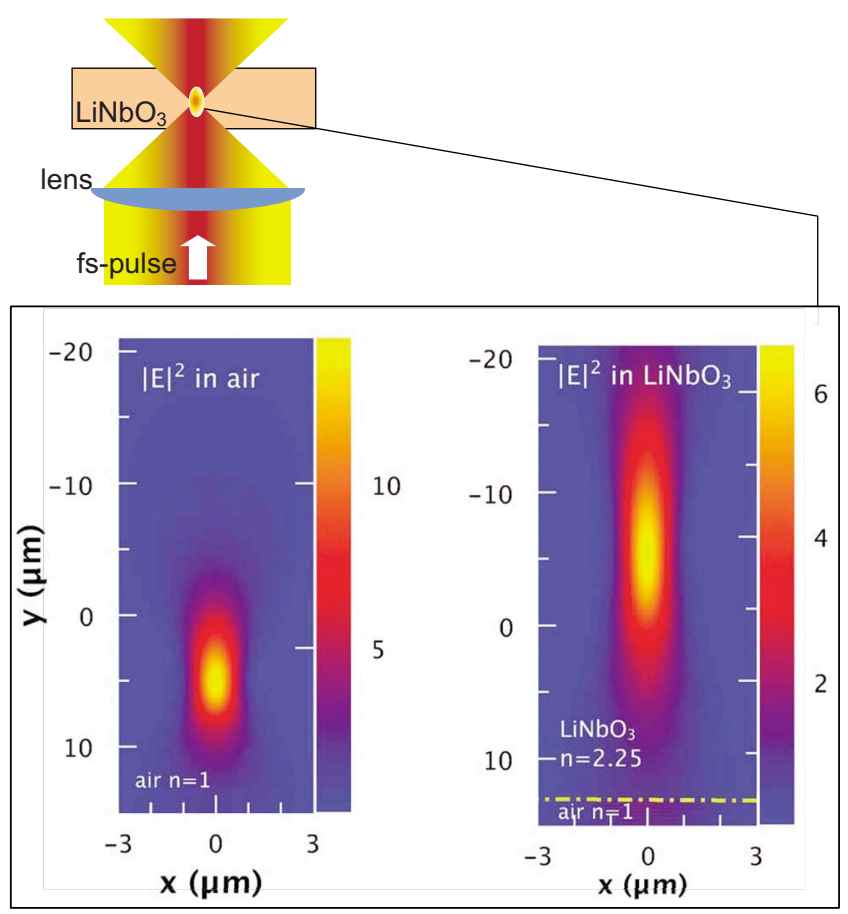

(b)

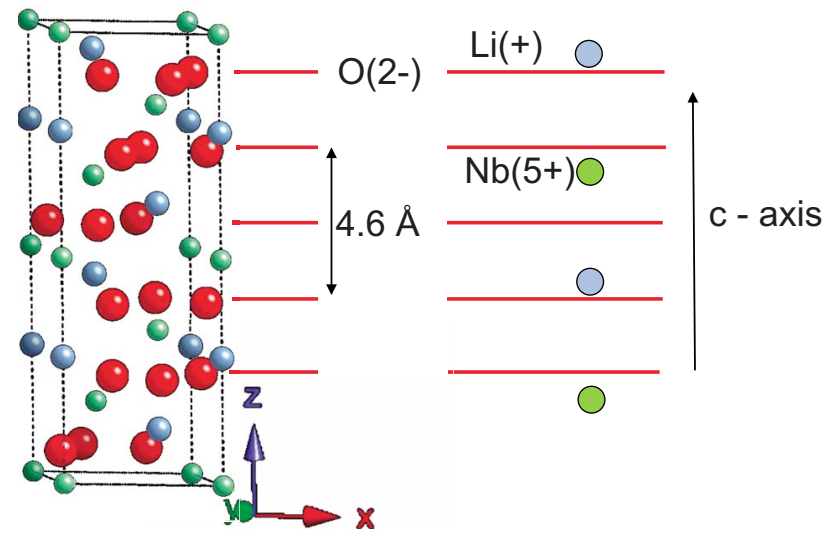

FIG. 1. (Color online) (a) The principle of fs laser structuring of photorefractive materials and the focal intensity distribution (inset). The light intensity for the aberration free (in air) and inside $\mathrm{LiNbO}_{3}$ focusing was simulated by a FDTD code for a focusing angle $\alpha=35^{\circ}(N A=n \sin \alpha)$, wavelength $800 \mathrm{~nm}$, and for a linearly polarized $E$ field $\mathbf{E}(1,0,0)$ of a Gaussian beam. The reflection from crystal-air boundary (the dashed line) reduced the intensity inside the crystal. (b) $\mathrm{LiNbO}_{3}$ cell structure [space group $R 3 m(161)$ (Ref. 21)] calculated by CRYSTALMAKER program.

used in our studies as described earlier. ${ }^{16} \mathrm{LiNbO}_{3}$ structure is the following: $\mathrm{Li}^{+}, \mathrm{Nb}^{5+}$, and $\mathrm{O}^{2-}$ as shown in Fig. 1(b). Band gap equals to $3.8 \mathrm{eV}$. In the doped crystal $\mathrm{LiNbO}_{3}: \mathrm{Fe}$ iron introduces a sublevel for $\mathrm{Fe}^{2+/ 3+}$ at $\sim 1.5 \mathrm{eV}$ below the conduction band. ${ }^{23,24}$ Iron concentration in our doped samples corresponds to the number density of irons of $18 \times 10^{18} \mathrm{~cm}^{-3}$. For more details see the Appendix. 


\section{PHOTOREFRACTIVE EFFECT AT DIFFERENT REGIMES}

In this section we revisit the conventional low-intensity approach to the photorefractive effect in order to demonstrate that this approach should be modified for the high-intensity case. The photovoltaic current, $j_{p h}$, excited in lithium niobate due to laser illumination with intensity $I$ is described in the simplified case of one-dimensional flow along optical axis by the following relation: ${ }^{25}$

$$
j_{p h}=\alpha G I,
$$

where $G$ is the Glass constant and $\alpha\left(\mathrm{cm}^{-1}\right)$ is inverse of the absorption length. It should be mentioned here that in this work we consider only the simplest single-center model of photorefractive medium. Hence, the higher-order contribution to the photovoltaic current as those observed in a quasi-cw high light-intensity regime ${ }^{26,27}$ are not taken into account. In fact, these contributions are only relevant for a $\mathrm{cw}$ or quasi-cw light illumination and hence do not play any role in discussed further single pulse excitation.

\section{A. Low intensity and long pulse}

The excitation rate of electrons (with density $n_{e}$ ) from valence to conduction band by the laser beam of low intensity, $I\left(\mathrm{~W} / \mathrm{cm}^{2}\right)$, is considered to be proportional to the number density of photons with the energy, $\hbar \omega$, arriving to the unit volume of an absorbing material per unit time, $\alpha I / \hbar \omega$. The excitation rate in accordance with Ref. 20 reads

$$
\frac{d n_{e}}{d t}=\Phi \frac{\alpha I}{\hbar \omega}
$$

here $\Phi$ is the quantum efficiency of a single photon, $\Phi=5 \times 10^{-4}$, and $\alpha$ is inverse of the absorption length (for lithium niobate $\alpha \sim 0.0033 \mathrm{~cm}^{-1}$ ) at a 800 -nm wavelength. ${ }^{28}$ One can see that the simple formula Eq. (2) is not applicable at high intensity. Indeed, let us consider lithium niobate affected by the $150 \mathrm{fs}, 800 \mathrm{~nm}(\hbar \omega=1.55 \mathrm{eV})$ laser pulse with the intensity of $10^{12} \mathrm{~W} / \mathrm{cm}^{2}$ that is close to the breakdown threshold. Taking the known material parameters, one obtains from Eq. (2) the number density of excited electrons at the end of the $150 \mathrm{fs}$ pulse equal to $\sim 10^{12} \mathrm{~cm}^{-3}$, that is nine orders of magnitude lower than critical electron density for $800 \mathrm{~nm}$ of $1.735 \times 10^{21} \mathrm{~cm}^{-3}$ that should correspond to the breakdown conditions. In Buse ${ }^{23}$ the excitation of electrons is presented as stationary process with excitation of electrons from $\mathrm{Fe}^{2+}$ centers (number density $n_{\mathrm{Fe}^{2+}}$ ) being balanced by recombination on $\mathrm{Fe}^{3+}\left(n_{\mathrm{Fe}^{3+}}\right)$ centers

$$
\frac{d n_{e}}{d t}=q S_{a b s} I n_{\mathrm{Fe}^{2+}}-\gamma n_{\mathrm{Fe}^{3+}} n_{e} .
$$

It is possible to reconcile source terms in Eqs. (2) and (3) by assuming that absorption occurs on $\mathrm{Fe}^{2+}$ centers, $\alpha=1 / l=n_{\mathrm{Fe}^{2+}} S_{a b s}$, where $S_{a b s}$ is the absorption cross section, and $q=\Phi / \hbar \omega$. In stationary case one obtains the number density as the following:

$$
n_{e}=\frac{q S_{a b s} I_{\mathrm{Fe}^{2+}}}{\gamma n_{\mathrm{Fe}^{3+}}} .
$$

The photovoltaic current is conventionally presented in the form

$$
j_{p h v}=e n_{e} v_{e} .
$$

The electron acceleration by the electric fields follows from the Newton equation

$$
m_{e} \frac{d v_{e}}{d t}=e E_{\text {las }}+e E_{\text {int }}-\nu_{e} m_{e} v_{e}
$$

Here, $\nu_{e}$ is the effective collision frequency responsible for resistance, $m_{e}$ is the electron mass, and $E_{\text {int }}$ represents all fields (including the photovoltaic, $E_{p h v}$ ) in a medium except for the incident laser field in the first term $\left(E_{\text {las }}\right)$. In the conventional photorefractive effect the electron motion is stationary and laser field is neglected. Thus the velocity of electron accelerated by the photovoltaic field $\left(E_{i n t}=E_{p h v}\right)$ reads

$$
v_{e}=\frac{e E_{p h v}}{\nu_{e} m_{e}}
$$

Now from Eqs. (1)-(7) one obtains the following expression for the photovoltaic field:

$$
E_{p h v}=\frac{G \gamma n_{\mathrm{Fe}^{3+}}}{q} \frac{\nu_{e} m_{e}}{e^{2}},
$$

which does not depend on the laser intensity but only on the material parameters. Hence this field must actually represent the field of spontaneous polarization that is an inherent property of ferroelectric crystals.

\section{B. High intensity and ultrashort pulse}

Three major mechanisms contribute to the light absorption in solids: ${ }^{29}$ the interband transitions (e.g., single and multiphoton absorption), intraband transitions (absorption on electrons in conduction band), and absorption on donor (acceptor) levels located inside the band gap.

It was found that the lowest light intensity enabling measurable diffractive-index structure formation by $150 \mathrm{fs}$ pulses is $\sim 4 \times 10^{11} \mathrm{~W} / \mathrm{cm}^{2}$ at $800 \mathrm{~nm}(6 \mathrm{~nJ}$ per pulse $)$. This intensity is just two to three times lower than the ionization threshold for the dielectrics. ${ }^{19}$ It is instructive to notice that the critical electron density, which signifies breakdown threshold, for $800 \mathrm{~nm}\left(\omega=2.35 \times 10^{15} \mathrm{~s}^{-1}\right)$ is $n_{c}=\frac{m_{e} \omega}{4 \pi e^{2}}=1.735 \times 10^{21} \mathrm{~cm}^{-3}$. The electric field amplitude at the intensity of $10^{12} \mathrm{~W} / \mathrm{cm}^{2}$ is $E=\sqrt{\frac{8 \pi I}{c}}=27.46 \mathrm{MV} / \mathrm{cm}$, that is two order of magnitude larger than the photovoltaic field of $100 \mathrm{kV} / \mathrm{cm}$ in lithium niobate..$^{25,30}$ The oscillation energy of electron reads ${ }^{31,32}$

$$
\varepsilon_{\text {osc }}=9.3\left[\frac{I}{10^{14}\left(\mathrm{~W} / \mathrm{cm}^{2}\right)}\right] \lambda_{\mu \mathrm{m}}^{2}(\mathrm{eV}),
$$

which for $1 \mathrm{TW} / \mathrm{cm}^{2}$ irradiance at $800 \mathrm{~nm}$ wavelength, yields $\varepsilon_{o s c}=0.06 \mathrm{eV}$, leading to the oscillation amplitude of 
3.9 A [for the scale see Fig. 1(b)]. This should be compared with the atomic displacements of $\mathrm{Li}$ of $0.9 \AA$ and $\mathrm{Nb}$ of $0.5 \AA$ in $\mathrm{LiNbO}_{3},{ }^{33}$ which are responsible for the spontaneous polarization. One may hence expect that during the laser pulse of high intensity the oscillating electrons affect the intrinsic crystal-field and ferroelectric properties. In these conditions one shall consider the major mechanisms, which are responsible for excitation of electrons to the conduction band from all constituent atoms present in the crystal. It is known that ionization by the electron impact (avalanche) and the ionization produced by simultaneous absorption of multiple photons are the two most important mechanisms of electron excitation in close to threshold conditions.

\section{Avalanche process}

A few (seed) electrons in the conduction band will oscillate in the electromagnetic field of the laser [Eq. (9)]. These electron can gain net energy by multiple electron-phonon (lattice) collisions and eventually be accelerated to reach the energy $\left(\varepsilon_{o s c}\right)$ in excess of the band gap $\varepsilon_{o s c}>\Delta_{\text {gap }}$. Energetic electrons create an avalanche of ionization events. The probability of such event per unit time (ionization rate) can be estimated as follows:

$$
w_{i m p} \approx \frac{\varepsilon_{\text {osc }}}{\Delta_{\text {gap }}} \frac{\omega^{2} \nu_{e-p h}}{\left(\nu_{e-p h}^{2}+\omega^{2}\right)} .
$$

Here $\nu_{e-p h}$, and $\omega$ are electron-phonon momentum exchange rate and laser frequency, respectively. Electron-phonon momentum exchange rate can be expressed as $\nu_{e-p h}^{(m o m)} \approx T_{L} / \hbar$ at the crystal temperature $\left(T_{L}\right)$ being in excess of the Debye temperature. ${ }^{29}$ At the room temperature of $293 \mathrm{~K}$ this rate equals to $3.83 \times 10^{13} \mathrm{~s}^{-1}$. For lithium niobate $\left(\Delta_{\text {gap }}\right.$ $=3.8 \mathrm{eV}$ ) under the action of 800-nm laser $\omega=2.35$ $\left.\times 10^{15} \mathrm{~s}^{-1} \gg \nu_{e-p h}\right)$ the avalanche excitation rate as function of laser intensity expresses then as $w_{\text {imp }} \approx 4.46$ $\times 10^{13} \frac{I}{10^{14}} \mathrm{~s}^{-1}$. Note that ionization rate of metal dopants is approximately twice higher because the doping introduces an additional energy level in the band gap.

\section{Multiphoton ionization}

It is reasonable to express the multiphoton ionization rate (probability of ionization per atom per second) in the form ${ }^{34}$

$$
w_{m p i} \approx \omega n_{p h}^{3 / 2}\left(\frac{\varepsilon_{\text {osc }}}{2 \Delta_{\text {gap }}}\right)^{n_{p h}},
$$

where $n_{p h}=\Delta_{\text {gap }} / \hbar \omega$ is the number of photons an electron should absorb in order to be transferred from valence to the conduction band. Again taking as an example lithium niobate under the action of 800-nm laser one gets the intensitydependent multiphoton ionization rate in the form

$$
w_{m p i} \approx 4.55 \times 10^{15}\left[\frac{I}{10^{14}}\left(\mathrm{~W} / \mathrm{cm}^{2}\right)\right]^{2.58}\left(\mathrm{~s}^{-1}\right) .
$$

\section{Number of electrons at the end of the pulse}

The number density of electrons $n_{e}$ created to the end of the pulse jointly, by the avalanche and multiphoton pro-
TABLE I. Electron-excitation parameters in lithium niobate evaluated for the $150 \mathrm{fs}$ pulse excitation at 800-nm wavelength evaluated using formulas (10)-(12) and (14).

\begin{tabular}{lcccc}
\hline \hline$I\left(\mathrm{~W} / \mathrm{cm}^{2}\right)$ & $4 \times 10^{11}$ & $10^{12}$ & $2 \times 10^{12}$ & $6 \times 10^{12}$ \\
\hline$\varepsilon_{\text {osc }}(\mathrm{eV})$ & 0.024 & 0.06 & 0.12 & 0.36 \\
$w_{\text {imp }}\left(\mathrm{s}^{-1}\right)$ & $0.18 \times 10^{12}$ & $0.45 \times 10^{12}$ & $0.9 \times 10^{12}$ & $2.7 \times 10^{12}$ \\
$w_{\text {mpi }}\left(\mathrm{s}^{-1}\right)$ & $2.96 \times 10^{9}$ & $3.15 \times 10^{10}$ & $1.88 \times 10^{11}$ & $3.2 \times 10^{12}$ \\
$n_{e}$ (at $\left.t=150 \mathrm{fs}\right)$ & $4.24 \times 10^{19}$ & $0.46 \times 10^{21}$ & $2.5 \times 10^{21}$ & $0.56 \times 10^{23}$ \\
\hline \hline
\end{tabular}

cesses, can be obtained with the help of the simplified rate equation $^{35}$

$$
\frac{d n_{e}}{d t}=n_{e} w_{i m p}+n_{a} w_{m p i},
$$

where $n_{a}$ denotes the atomic density. Let us assume that laser intensity is constant during the laser pulse (a flat-top intensity distribution). As we show later the recombination during the pulse time can be neglected. Then the solution to Eq. (2) with the initial condition $n_{e}(t=0)=n_{e 0}$ and $w_{i m p}$ and $w_{m p i}$ (both time independent) is straightforward

$$
n_{e}(I, \lambda, t)=\left\{n_{e 0}+\frac{n_{a} w_{m p i}}{w_{\text {imp }}}\left[1-\exp \left(-w_{\text {imp }} t\right)\right]\right\} \exp \left(w_{\text {imp }} t\right) .
$$

It is commonly accepted that breakdown of a dielectric occurs when the plasma frequency of excited electrons equals to the frequency of impinging laser light. Intensitydependent oscillation energy, ionization rates, and number density of excited electrons on the basis of formulas (9)-(13) to the end of $150 \mathrm{fs}, 800 \mathrm{~nm}$, laser pulse in lithium niobate are presented in the Table I and plotted in Fig. 2. The critical electron density at $800 \mathrm{~nm}$ is $1.735 \times 10^{21} \mathrm{~cm}^{-3}$. Thus one can conclude that the above simple model predicts the breakdown threshold to be around $1.5 \times 10^{12} \mathrm{~W} / \mathrm{cm}^{2}$ in semi-

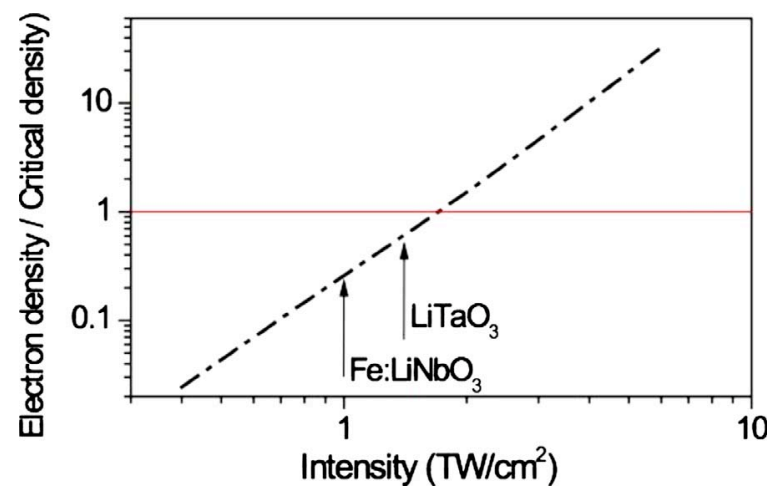

FIG. 2. (Color online) Normalized electron density vs laser intensity at $800-\mathrm{nm}$ wavelength (Table I); focusing by a lens of numerical aperture $N A=0.55$ ); the horizontal line marks the critical density. The optically detectable changes in refractive index in $\mathrm{Fe}: \mathrm{LiNbO}_{3}$ and undoped $\mathrm{LiTaO}_{3}$ are marked by arrows at 1 and $1.37 \mathrm{TW} / \mathrm{cm}^{2}$, respectively. 
quantitative agreement with reported earlier experiments in glass. ${ }^{19}$

\section{Recombination rate and time}

Recombination in the three body collisions may occur with electron, ion, or neutral atom all acting as a third body. One can take an atomic cross section for estimation of $e-i$ collision rate in the case when ionization degree is below the breakdown threshold as in the considered here case. The probability of recombination per unit volume per unit time (recombination rate) is then a product of probability of $e-i$ collision, $\nu_{c o l}=n_{e} n_{+} \sigma_{o} v_{e}$, (where $n_{+}$and $\sigma_{o}$ denote the ion concentration and the recombination cross section, respectively), and the probability for a third body presence in the vicinity of colliding particles, $p_{3 b}$. The latter factor can be approximated by the ratio of atomic volume to the average volume per atom, $p_{3 b} \approx 4 / 3 \pi r_{a t}^{3} n_{a}$, if an atom can be considered as a third body. Here $r_{a t}$ and $n_{a t}$ denote atomic radius and atomic density, respectively. In this case this probability is close to unity. Thus recombination time in below-threshold conditions $\left(n_{e}<1.735 \times 10^{21} \mathrm{~cm}^{-3}\right)$ is estimated to be $t_{r e c} \approx\left(n_{e} \sigma_{a t} v_{e}\right)^{-1} \sim\left(10^{21} \times 10^{-15} \times 10^{6}\right)^{-1}$ and it is around 1 ps.

At the electron density close to the breakdown threshold the recombination in the triple Coulomb collisions when the electron acts as a third body can be of importance. In this case the Coulomb collisions are characterized by the cross section $\pi r_{0}^{2} \approx \pi\left(e^{2} / \varepsilon_{e l}\right)^{2}$. Here $\varepsilon_{e l}$ is the electron energy. Excited electrons in the conduction band can be treated as the degenerated Fermi gas with the energy $\varepsilon_{F}=\left(3 \pi^{2} n_{e}\right)^{2 / 3} \frac{\hbar^{2}}{2 m_{e}}$, Fermi velocity $\left(v_{F}=2.5 \times 10^{7} \mathrm{~cm} / \mathrm{s}\right)$ and heat capacity $C_{e}=\pi^{2} T_{e} /\left(2 \varepsilon_{F}\right)$. At $n_{e}=10^{21} \mathrm{~cm}^{-3} \varepsilon_{e l} \approx \varepsilon_{F}=0.36 \mathrm{eV}$. One can easily see that the Coulomb cross section is two orders of magnitude higher than the atomic cross section. The electron mean-free path $l_{m f p}=v_{F} / \nu_{e-p h} \sim 10^{-6} \mathrm{~cm}$ is much shorter than the laser-modified absorption length as it is shown below. Therefore the recombination time in the triple Coulomb collisions can be on the order of femtoseconds and hence the recombination during the pulse time might be significant and fast. On the other hand, the recombination time is inversely proportional to the electron density. Taking recombination cross section and electron velocity as time independent one obtains, $t_{\text {rec }} \approx t_{0} n_{0} / n_{e}$, and very slow decrease in time for the electron number density, $n_{e}(t)=n_{e}\left(t_{p}\right) t_{0} / t$. Here $t_{0}$ is recombination time at the end of the pulse. For $n_{e}\left(t_{p}\right)=10^{21} \mathrm{~cm}^{-3}$ one gets $t_{0}=1 \mathrm{ps}$. It is clear that the electron number density becomes comparable to that for metal dopants, of $\sim 10^{18} \mathrm{~cm}^{-3}$ in nanosecond time as it was suggested at lowintensity studies. ${ }^{20}$

\section{Modification of material optical properties to the pulse end}

Let us now consider the change in the optical properties of the medium during the pulse time under assumption that light intensity during the pulse is lower than the breakdown (damage) threshold and therefore the laser-induced modification could be reversible. The total dielectric function for a dielectric modified by electrons excitation at high intensity is the sum of unperturbed function $\left(\epsilon_{0}\right)$ and contributions from excited electrons

$$
\begin{aligned}
\epsilon & =\epsilon_{0}(\omega)+i \frac{4 \pi \sigma}{\varepsilon_{0} \omega} \\
& \equiv \epsilon_{0}(\omega)-\frac{4 \pi \sigma_{i m}}{\varepsilon_{0} \omega}+i \frac{4 \pi \sigma_{r e}}{\varepsilon_{0} \omega} \\
& \equiv \epsilon_{0}(\omega)-\Delta \epsilon_{r e}+i \Delta \epsilon_{i m},
\end{aligned}
$$

where $\sigma_{r e, i m}$ are the real and imaginary parts of conductivity, respectively.

It is reasonable to take the dielectric function and conductivity for excited electrons in the Drude form

$$
\sigma=\frac{e^{2} n_{e}}{m_{e}\left(\nu_{e f f}-i \omega\right)}=\frac{e^{2} n_{e} \nu_{e-p h}}{m_{e}\left(\nu_{e-p h}^{2}+\omega^{2}\right)}+i \frac{e^{2} n_{e} \omega}{m_{e}\left(\nu_{e-p h}^{2}+\omega^{2}\right)} .
$$

Then one can find the contributions to the real and imaginary parts of $\epsilon$ at $\omega \gg \nu_{e-p h}$ as follows

$$
\Delta \epsilon_{r e} \approx \omega_{p e}^{2} / \omega^{2}, \quad \Delta \epsilon_{i m} \approx \omega_{p e}^{2} \nu_{e-p h} / \omega^{3} ; \quad \sigma_{r e}=\omega \Delta \epsilon_{i m} / 4 \pi,
$$

where $\omega_{p e}^{2}=4 \pi e^{2} n_{e} /\left(\varepsilon_{0} m_{e}\right)$ is the squared plasma frequency. Note that in considered here conditions the following inequality holds, $\Delta \epsilon_{i m} \ll \Delta \epsilon_{r e}$. The modified refractive index, $N \equiv \sqrt{\epsilon}=n+i k$, then reads

$$
n \approx\left(\epsilon_{0}-\Delta \epsilon_{r e}\right)^{1 / 2} ; \quad k \approx \Delta \epsilon_{i m} / 2 n .
$$

Note that the change in the refractive index due to electron excitation is negative

$$
-\Delta n / n_{0} \approx \Delta \epsilon_{r e} / 2 n_{0}^{2} .
$$

\section{Electron and lattice temperature}

Now all-important characteristics of the laser-excited crystal such as the absorption length, $l_{s}=\frac{c}{\omega k}$, the absorption coefficient from the Fresnel formula $A \approx \frac{4 n}{(n+1)^{2}}(n \gg k)$, the absorbed energy density, $E_{a b s}=\frac{2 A F_{p}}{l_{s}}$, to the end of a $150 \mathrm{fs}$ laser pulse can be calculated. We assume that electrons in conduction band can be considered in the degenerate state and therefore to be characterized by the Fermi energy $\varepsilon_{F} \propto n_{e}^{2 / 3}$ and the corresponding heat capacity. Under these assumptions the electron temperature (considered as the energy in the excess of the Fermi level) can be calculated as $T_{e m}^{2}=\frac{4}{\pi^{2}} \frac{\varepsilon_{F} A F_{p}}{n_{e} l_{s}}$.

To obtain numerical estimates let us assume that the number density of excited electrons is in a range $10^{20}-10^{21} \mathrm{~cm}^{-3}<n_{c}$, lower but in the vicinity of the critical density. Therefore squared plasma frequency is in a range $\omega_{p e}^{2}=(0.318-3.18) \times 10^{30} \mathrm{~s}^{-2}$, of the same order of magnitude as the squared laser frequency, $5.52 \times 10^{30} \mathrm{~s}^{-2}$ and both are much higher than the electron-phonon momentum exchange rate. The absorbed energy density to the end of the pulse, $F_{p}$, is taken as the average intensity by the pulse duration $F_{p}=I \times t_{p}$. Dielectric function of unperturbed lithium 
TABLE II. Modified optical properties, absorbed energy, and temperature of 150 fs excited lithium niobate crystal.

\begin{tabular}{|c|c|c|c|c|c|c|c|c|c|}
\hline$n_{e}$ & $\Delta \epsilon_{r e}$ & $\Delta \epsilon_{i m}$ & $\Delta n / n_{0}$ & $k \approx \Delta \epsilon_{i m} / 2 n$ & $\begin{array}{c}l_{s} \\
(\mathrm{~cm})\end{array}$ & $A$ & $\begin{array}{c}E_{a b s} \\
\left(\mathrm{~J} / \mathrm{cm}^{3}\right)\end{array}$ & $\begin{array}{c}T_{e m} \\
(\mathrm{eV})\end{array}$ & $\begin{array}{c}\sigma_{r e} \\
10^{12}\left(\mathrm{~s}^{-1}\right)\end{array}$ \\
\hline $10^{20}$ & 0.057 & $0.94 \times 10^{-3}$ & -0.0056 & $0.193 \times 10^{-3}$ & $6.6 \times 10^{-2}$ & 0.82 & & & 0.176 \\
\hline $10^{21}$ & 0.576 & $9.4 \times 10^{-3}$ & -0.056 & $2.0 \times 10^{-3}$ & $6.4 \times 10^{-3}$ & 0.84 & 39.4 & 0.28 & 1.76 \\
\hline
\end{tabular}

niobate at low intensity at $800 \mathrm{~nm}$ equals to $\epsilon_{0}=5$ (the ordinary refractive index $n=2.254){ }^{28}$

The characteristics of the laser-modified crystal at the intensity of $1.5-0.55 \mathrm{TW} / \mathrm{cm}^{2}$ at $800 \mathrm{~nm}$ wavelength estimated using the formulas given above are presented in the Table II and illustrated in Fig. 2.

Note that intense laser pulse drastically modifies the optical properties. For example, the effective absorption that defines the absorbed energy density equals to $A / l_{s}=131 \mathrm{~cm}^{-1}$ at average intensity during the pulse around TW that should be compared to $\alpha \sim 0.0033 \mathrm{~cm}^{-1}$ (for $800 \mathrm{~nm}$ ) for a low intensity long pulse case. The absorbed energy density at $I \times t_{p}=0.15 \mathrm{~J} / \mathrm{cm}^{2}$ reaches $39.4 \mathrm{~J} / \mathrm{cm}^{3}$. This may result in only insignificant increase in the lattice temperature. Maximum change in the refractive index due to excitation of electrons is negative on the order of $10^{-2}$ that is much larger than the modification due to photovoltaic field at low intensity. However, this modification decreases fast after the end of the pulse due to recombination. In principle, it could be detected with pump-probe technique.

\section{Relaxation processes in the laser-excited photorefractive crystal}

As it follows from the Table II the excited electrons gain significant energy to the end of the short pulse. Their energy distribution is close to that of the equilibrium Fermi gas. However the absorbed energy density is not high enough to induce melting or other structural changes. The electron-tolattice energy-transfer time can be estimated as the following:

$$
\nu_{e-p h}^{e n} \approx\left(\frac{m_{e}}{M}\right)^{1 / 2} \nu_{e-p h}^{(m o m)},
$$

where $M$ denotes the average atomic mass. For lithium niobate $(M=29.57)$ this rate is $1.63 \times 10^{11} \mathrm{~s}^{-1}$, giving the lattice heating time of $\sim 6 \mathrm{ps}$. Thus the lattice reaches the maximum temperature rise during this time taking into account that heat conduction in $\mathrm{LiNbO}_{3}$ is poor (heat-diffusion coefficient is $D=1.5 \times 10^{-2} \mathrm{~cm}^{2} / \mathrm{s}$ ). One can also observe that cooling of micron-size laser-affected spot, $d$, takes time of $t_{\text {cool }}=d^{2} / D \sim 700 \mathrm{~ns}$. Assuming that heat capacity obeys the Dulong-Petit law one can estimate the lattice temperature increase as the following:

$$
T_{L, \max }=\left(\frac{2 A F_{p}}{l_{s}}\right) / C_{L} n_{a} .
$$

Thus, the lattice temperature of lithium niobate increases by $10 \mathrm{~K}$ for the absorbed energy density of $39.4 \mathrm{~J} / \mathrm{cm}^{3}$. One cannot expect any structural changes at such temperature. However the temperature rise will induce the positive tran- sient index change on the order of $10^{-4}$ via the thermo-optic effect. ${ }^{36}$ It also follows from the above that the cooling during the time necessary for establishing a quasistationary distribution of charges can be neglected.

\section{E. Quasistationary optical changes after fs pulse}

As we demonstrated above, the conditions in photorefractive crystal created by the action of intense femtosecond pulse are in sharp contrast to those produced by long lowintensity pulse or $\mathrm{cw}$ lasers. First all processes during the pulse are transient. The quasistationary state is achieved long after the pulse end. We shall consider now all relevant physical processes contributing toward the optical changes in the material during and after the pulse.

\section{Processes during the pulse}

Let us estimate the electron current, $j_{p h v}=e n_{e} v_{e}$, that might be created during the short (150 fs) and intense $\left(10^{12}-10^{13} \mathrm{~W} / \mathrm{cm}^{2}\right)$ pulse. The pulse generates the number of excited electrons on the order of $10^{21} \mathrm{~cm}^{-3}$. The electrons during the pulse oscillate in the high-frequency electric field with amplitude $(2.7-8.7) \times 10^{7} \mathrm{~V} / \mathrm{cm}$ several orders of magnitude higher than the inherent field of spontaneous polarization in photorefractive crystals or photovoltaic field induced in a crystal. Therefore it is impossible to establish a stationary distribution during the pulse time.

Photoconductivity obtained from Eq. (15) (assuming that the collision rate can be neglected in comparison to the laser frequency) reads

$$
\sigma_{p h} \approx \frac{e^{2} n_{e} \nu_{e-p h}}{m_{e} \omega^{2}}
$$

and it is a strong function of the carrier number density. For $800-\mathrm{nm}$ light $\left(\omega=2.356 \times 10^{15} \mathrm{~s}^{-1}\right)$, and collision rate of $3.8 \times 10^{13} \mathrm{~s}^{-1}, \quad n_{e}=10^{21} \mathrm{~cm}^{-3}$ Eq. (21) yields $\sigma_{p h} \sim 2 \Omega^{-1} \mathrm{~cm}^{-1}\left(1.74 \times 10^{12} \mathrm{~s}^{-1}\right)$. The above conductivity value defines the time for establishing quasistationary distribution of electric field, $t_{\text {stat }} \approx \varepsilon_{s t} /\left(4 \pi \sigma_{p h}\right) \sim 1 \mathrm{ps}$, where $\varepsilon_{s t}$ is the static (in the absence of external field) dielectric function. Thus stationary distribution can be established only after the end of the pulse.

\section{Processes after the pulse}

After the pulse end the electrons are subject of recombination, drift in a local field of spontaneous polarization inherent to a photorefractive crystal, diffusion under the electrons temperature and density gradients, while ions remain fixed. Diffusion field is much smaller than other fields and it 
can be neglected. Essential difference with conventional case (long pulses or cw illumination) is that the field of charge separation which is finally responsible for quasipermanent change in the refractive index, establishes in the absence of external field or irradiation due to the spontaneous polarization field. Because the recombination time is longer than the pulse duration, the carriers recombine in a different location from those where they were created, most probably in the iron sites. Thus we can assume that the charge separation field, $E_{c s}$, equals to $E_{s}$ - the internal field of spontaneous polarization in the ferroelectric crystal supposedly unaffected by the electron excitation. It is conventionally assumed that quasistationary distribution establishes during the so-called Maxwell time s7 $^{37}$

$$
t_{\text {stat }} \approx \varepsilon_{s t} / 4 \pi \sigma_{d} .
$$

The conductivity in the absence of photoexcitation (the socalled dark conductivity) reads

$$
\sigma_{d} \approx \frac{e^{2} n_{e}}{m_{e} \nu_{e-p h}} .
$$

Note that $\sigma_{d} \gg \sigma_{p h}$ because $\omega \gg \nu_{e-p h}$. Taking $\varepsilon_{s t}=29$; $n_{e} \sim 10^{18} \mathrm{~cm}^{-3}, \nu_{e-p h}^{(m o m)} \approx T_{L} / \hbar=3.83 \times 10^{13} \mathrm{~s}^{-1}$, and supposing that electron mass equals to that for a free electron, the time for establishing the stationary distribution is less than a picosecond.

The inherent electric field in a crystal due to spontaneous polarization is

$$
E_{s}=\frac{P_{s}}{3 \varepsilon_{0}},
$$

where $\varepsilon_{0}$ is the permittivity of free space. Following Glass $e t$ $a l .{ }^{30}$ this field in the lithium niobate is on the order of $E_{s}$ $=100 \mathrm{kV} / \mathrm{cm}$.

\section{Refractive-index changes after the pulse}

Generation of large amount of excited electrons immediately results in the decrease in the refractive index [see, Eqs. (16) and (18)]

$$
-\Delta n / n_{0} \approx \Delta \epsilon_{r e} / 2 n_{0}^{2} \approx \omega_{p e}^{2} / 2 n_{0}^{2} \omega^{2}
$$

and at the number density to the pulse end of $n_{e}=10^{21} \mathrm{~cm}^{-3}$ constitutes $\Delta n / n_{0}=-5.6 \times 10^{-2}$ (see Table II). This index modification decreases in proportion to the decreasing number density of free carriers due to recombination and reaches the level of $\Delta n / n_{0} \sim 5 \times 10^{-5}$ over the $\sim$ nanosecond time scale.

The change in the refractive index due to the electro-optic effect reads ${ }^{37}$

$$
\Delta n \approx \frac{n^{3} r}{2} E_{c s}
$$

Taking the known value of $n^{3} r \approx 3 \times 10^{-8} \mathrm{~cm} / \mathrm{V}$ for lithium niobate (for extraordinary polarized reading light) one can estimate the refractive-index change $\Delta n \approx 1.5 \times 10^{-8}(\mathrm{~cm} / \mathrm{V}) \times E_{c s}$. For $E_{c s} \sim 10^{2} \mathrm{kV} / \mathrm{cm}$ this index change is expected to be on the order of $\Delta n \approx 1.5 \times 10^{-3}$.

\section{F. Comparison with experiments}

Interaction of single femtosecond laser pulses tightly focused in nominally pure and Fe-doped $\mathrm{LiNbO}_{3}$ was recently studied and reported in the literature. ${ }^{8,16,17}$ Single shots of 150 fs laser pulses with wavelength $800 \mathrm{~nm}$, energy per pulse in a range of 3-50 $\mathrm{nJ}$, were focused inside a crystal to the depth of $50 \mu \mathrm{m}$ to the focal spot with diameter of $1.8 \mu \mathrm{m}$ (with area of $2.54 \times 10^{-8} \mathrm{~cm}^{2}$ ). Thus the intensity in the interaction region varied from $1 \mathrm{TW} / \mathrm{cm}^{2}$ to 16.7 $\mathrm{TW} / \mathrm{cm}^{2}$. The threshold for optically detectable change in refractive index has been found in terms of energy per pulse to be $3.8 \pm 0.5 \mathrm{~nJ}$ in $\mathrm{Fe}: \mathrm{LiNbO}_{3}$ and $5.2 \pm 0.5 \mathrm{~nJ}$ in pure $\mathrm{LiNbO}_{3}$ crystal (Fig. 2; based on calculations presented in Table I). These figures correspond to intensity of $1 \mathrm{TW} / \mathrm{cm}^{2}$ and $1.37 \mathrm{TW} / \mathrm{cm}^{2}$, respectively, and are below the threshold of critical plasma density. The permanent modification of $\mathrm{LiTaO}_{3}$ was observed at $32 \pm 5 \mathrm{~nJ}$ $\left(\sim 10 \mathrm{TW} / \mathrm{cm}^{2}\right)$ that might be considered as a result of dielectric breakdown. Therefore the breakdown threshold locates at $\leq 10 \mathrm{TW} / \mathrm{cm}^{2}$. It also has been found that the laserinduced material transformation is fully reversible at the energy per pulse of $14.5 \mathrm{~nJ}\left(3.8 \mathrm{TW} / \mathrm{cm}^{2}\right)$. One can see that our theoretical estimates of breakdown are in qualitative agreement with the measurements. The actual energy density at the focus is expected to be smaller due to the presence of spherical aberration which is proportional to the refractiveindex mismatch between surrounding medium (immersion oil $n=1.515)$ and the photorefractive crystal $(n \approx 2.25)$ and the focusing depth, as was observed in glass at similar focusing conditions. ${ }^{38}$ It is worth to note that one can compensate for the spherical aberration by using the wave-front correction techniques. ${ }^{22}$ Thus it is confirmed that generation of free carriers by intense fs pulses occurs due to intertwined avalanche and multiphoton processes.

The changes in refractive index were recovered by measuring diffraction efficiency of linearly polarized light beams $(\lambda=632.8 \mathrm{~nm})$ illuminating periodic index pattern (period $3 \mu \mathrm{m}$ ) formed with our single femtosecond pulses. In $\mathrm{Z}$-cut pure $\mathrm{LiTaO}_{3}$ crystal the maximum measured index change was $2.5 \times 10^{-4}$. In a Y-cut nominally pure $\mathrm{LiNbO}_{3}$ the index change was $5 \times 10^{-4}$ (for the extraordinary polarization of the reading beam). Maximum reported reversible index change in $\mathrm{Fe}: \mathrm{LiNbO}_{3}$ (400 ppm doping) reached $\sim 10^{-3}$. The image contrast of the laser-affected area of the crystal exhibits a characteristic dark/bright/dark region (as compared to a homogeneous background) along the crystallographic $c$ axis. This occurs in both, Fe-doped and undoped crystals. Such contrast variation must correspond to the refractive-index modulation pattern " $+\Delta n /-\Delta n /+\Delta n$ " where the plus (minus) sign reflects index increase (decrease) with respect to its initial value. While we have not measured the index modulation interferometrically, it is well established that in lithium niobate such contrast and index patterns result from the action of photovoltaic field which decreases refractive index in the central part of the illuminated spot. ${ }^{8,39}$ There is however a substantial difference in the after-the-pulse behavior of laseraffected regions in Fe-doped and undoped $\mathrm{LiNbO}_{3}$. Index modulation in Fe-doped crystal is long lived while in undoped crystal it completely disappears after $0.25-0.3 \mathrm{~s}$, most 


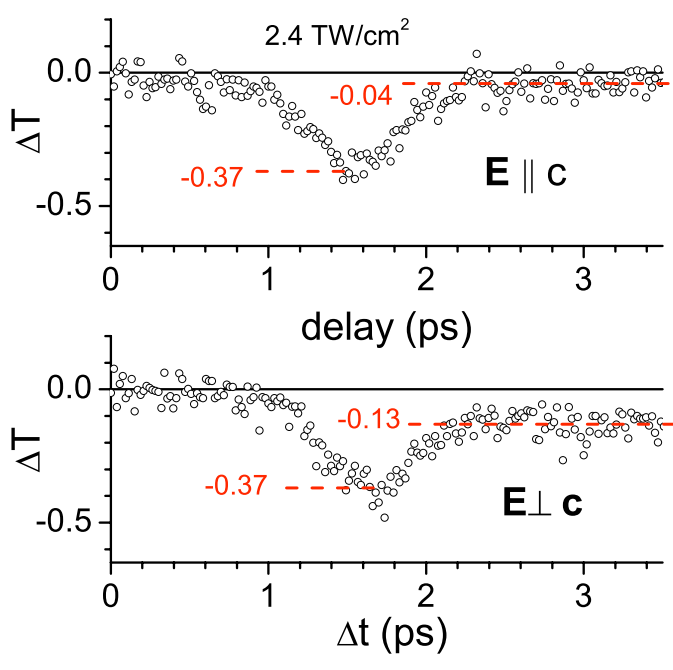

FIG. 3. (Color online) Transient differential transmission of undoped $\mathrm{LiNbO}_{3}$ for the same pump intensity and two different orientations of pump polarization with respect to $c$ axis.

probably due to full recombination of the free carriers. On the other hand the long life of the charge separation and resulted modulation of refractive index in Fe-doped crystal occurs due to the presence of the trapping centers $\left(\mathrm{Fe}^{3+}\right)$ separated by distance of $1 / n^{1 / 3} \approx 10^{-6} \mathrm{~cm}$ from each other, which is comparable to the maximum free-carrier mean-free path. The last fact indicates that the nonlocal approach should be applied for the current calculation instead of the Ohm's law.

Transient transmission in undoped Y-cut samples of $\mathrm{LiNbO}_{3}$ was studied by femtosecond pump-probe technique. In the experiments, femtosecond Ti:Sapphire amplified laser with a central wavelength of $800 \mathrm{~nm}$ and a pulse duration of 150 fs was used. Intense pulses of fundamental wavelength served as a pump while weaker frequency-doubled pulses with a central wavelength of $400 \mathrm{~nm}$ were used as a probe. The pulses were focused into $\mathrm{LiNbO}_{3}$ using a microscope lens with numerical aperture of 0.45 at the depth of approximately $40-50 \mu \mathrm{m}$ below the surface of samples whose total thickness was $1 \mathrm{~mm}$, much longer than the waist of the focused pump beam. The probe beam was expanded prior to the focusing lens to produce focal spot smaller than that of the pump beam in order to probe a region more uniformly excited by the pump. The pump and probe beams were polarized linearly and their polarization planes were mutually orthogonal. Intensity of the pump pulse at the focus of $2.3 \mathrm{TW} / \mathrm{cm}^{2}$ was determined from the single pulse energy and area of the focal spot but this value is likely overestimated because focusing aberrations were ignored. Figure 3 shows transient differential transmission at the same pump pulse intensity and pump polarization parallel and perpendicular to the crystallographic $c$ axis. Probe differential transmission $\Delta T=\left(I-I_{0}\right) / I_{0}$, where $I_{0}$ and $I$ are transmitted probe intensities in the absence and presence of the pump beam, respectively, was measured as a function of temporal delay $\Delta t$ between the pump and probe pulses. Due to experimental difficulty in establishing the absolute position of zero pumpprobe delay, the origin of time delay axis in the figure is chosen arbitrarily; the actual $\Delta t=0$ is most likely close to the points where transmission becomes lowest. The measured transmission transients exhibit sharp dips near the expected zero delay. Temporal width of these dips is comparable to (albeit slightly larger than) the pulse duration. This fast darkening indicates direct band-to-band excitation of $\mathrm{LiNbO}_{3}$ via multiphoton absorption during the action of pump pulse. The fast parts are followed by plateaus, which in fact mark the beginning of very slow relaxation extending to milliseconds, as confirmed by in situ observation of the optically pumped region conducted during the measurements. A visible darkening of the spot could be seen and its relaxation could be roughly followed within few hundreds of milliseconds at a rate provided by an ordinary video camera. These slow parts can be assigned to absorption of the probe by photoexcited carriers. According to Fig. 3, the fast parts are independent of the orientation of polarization with respect to $c$ axis whereas the plateau is slightly deeper for the case of $\mathbf{E} \perp c$. According to the literature ${ }^{40,41}$ this difference occurs due to orientation of the probe polarization. In general, the results shown in Fig. 3 are similar to the results of pump-probe measurements reported elsewhere ${ }^{40,41}$ despite the use of collinear pumpprobe and $\mathrm{Fe}$-doped samples in the latter case.

\section{DISCUSSION AND CONCLUSIONS}

We demonstrated theoretically that the interaction of intense femtosecond pulse with photorefractive crystal at conditions close to the breakdown threshold has several distinctive features in comparison to that of long pulse (or $\mathrm{cw}$ ) lasers. First, we showed that the high number density of excited electrons, two orders of magnitude higher than number density of dopants, modifies the dielectric function and leads to the negative change in refractive index, $\sim 10^{-2}$, exceeding that due to the charge separation long after the end of the pulse. This change depends on laser intensity and it is independent of polarization. However, this index change is transient and it disappears when the recombination is completed at the nanosecond time scale.

Second, the dominance of high-frequency laser field that is two orders of magnitude higher than the field of spontaneous polarization makes impossible the stationary charge distribution during the pulse. Third, diffusion and recombination of charge carriers continue long after the end of the pulse (on the nanosecond time scale). The main driving force responsible for the current is the field of spontaneous polarization: the current terminates when the field of charge separation balances this field. Quasistationary distribution of charges that results in change in the refractive index on the order of $10^{-3}$ due to the Pockels effect occurs well after the end of the pulse. Modification of refractive index derived from this model is in a semiquantitative agreement with experiment. As the quasistationary modification of refractive index takes place long after the pulse it is independent of the polarization of the writing beam-in agreement with experimental observations. ${ }^{8,19}$

Our findings suggest that the high-amplitude laser electric field modifies not only linear properties of the material. Most probably strong ac field also induces transient ferroelectric and nonlinear properties of a crystal. Therefore an avenue 
opens up for the studies of the intensity-dependent transient phase transformation induced by femtosecond laser at intensity close but below the damage threshold. Pump-doubleprobe experiments might provide the information of timedependent dielectric function of excited crystal with fs resolution while harmonic generation may provide information on transient nonlinear properties.

\section{ACKNOWLEDGMENTS}

Financial support provided by the Australian Research Council Discovery Grant and a Grant-in-Aid from the Ministry of Education, Science, Sports, and Culture of Japan under Grant No. 19360322 are gratefully acknowledged. We also acknowledge discussions with Kenji Kitamura.

\section{APPENDIX}

Here, we collect parameters of lithium niobate ${ }^{28}$ and some of the used estimations. The cooling time is estimated as the following. Thermal conductivity $\kappa=5.6 \times 10^{-2} \mathrm{~W} / \mathrm{cm} \mathrm{K}$; and $D=\kappa / c_{p} n_{a}=1.7 \times 10^{-2} \mathrm{~cm}^{2} / \mathrm{s}$. The cooling time for micron-size area comprises $t_{\text {cool }}=10^{-8} / 1.7 \times 10^{-2} \mathrm{~cm}^{2} / \mathrm{s}$ $=590$ ns (Table III).

Band gap: ${ }^{28} \quad E_{g}=3.9-4.3 \mathrm{eV}$ (direct) and $E_{g}=3.3 \mathrm{eV}$ (indirect). Position of sublevel in the band gap due to
TABLE III. Material and optical properties of lithium niobate (Ref. 28).

\begin{tabular}{lc}
\hline \hline Chemical formula & $\mathrm{LiNbO}_{3}$ \\
\hline Band gap, eV & 4.0 \\
Melting temperature, $\mathrm{K}$ & 1530 \\
Curie temperature, $\mathrm{K}$ & $1415(1480)$ \\
Dielectric constant at $100 \mathrm{KHz}$ & 85,29 \\
$\epsilon_{a}$ (perpendicular), $\epsilon_{c}$ (parallel) & \\
Density, g/cm ${ }^{3}$ at $293 \mathrm{~K}$ & 4.644 \\
Thermal conductivity, W/(m K) at $300 \mathrm{~K}$ & 5.6 \\
Specific heat, cal/(g K) & 0.15 \\
Transmittance range, nm & $350-5500$ \\
\hline \hline
\end{tabular}

doping by iron: approximately $2.5 \mathrm{eV}$ from the conduction band. ${ }^{24}$ The quantum efficiency for exciting of charge carriers $\Phi=5 \times 10^{-4}$. Optical properties: ${ }^{28}$ at $\lambda=800 \mathrm{~nm}$ $\left(\omega=2.356 \times 10^{15} \mathrm{~s}^{-1} ; 1.55 \mathrm{eV}\right)$, ordinary refractive index $n=2.254$ (at $\lambda=800 \mathrm{~nm}$ ) and 2.286 (at $\lambda=632.8 \mathrm{~nm}$ ).

The binding energy for the lithium niobate can be estimated through the phonon frequency $\omega_{p h}$, average mass per atom $M_{a v}$, and nearest neighbor distance $d$ as follows: $\epsilon \simeq M_{a v} \omega_{p h}^{2} d^{2} \approx 4.5 \mathrm{eV} /$ atom. *gam111@rsphysse.anu.edu.au

${ }^{1}$ K. Buse, A. Adibi, and D. Psaltis, Nature (London) 393, 665 (1998).

${ }^{2}$ A. Adibi, K. Buse, and D. Psaltis, J. Opt. Soc. Am. B 18, 584 (2001).

${ }^{3}$ N. V. Kukhtarev, G. E. Dovgalenko, and V. N. Starkov, Appl. Phys. A A33, 227 (1984).

${ }^{4}$ B. Sturman, M. Carrascosa, and F. Agullo-Lopez, Phys. Rev. B 78, 245114 (2008).

${ }^{5}$ N. Kukhtarev, V. Markov, and S. Odulov, Zh. Tekh. Fiz. 50, 1905 (1980).

${ }^{6}$ Y. Kawata, H. Ishitobi, and S. Kawata, Opt. Lett. 23, 756 (1998).

${ }^{7}$ G. Zhou and M. Gu, Appl. Phys. Lett. 87, 241107 (2005).

${ }^{8}$ S. Juodkazis, M. Sudzius, V. Mizeikis, H. Misawa, E. G. Gamaly, Y. Liu, O. A. Louchev, and K. Kitamura, Appl. Phys. Lett. 89, 062903 (2006).

${ }^{9}$ G. Zhou and M. Gu, Opt. Lett. 31, 2783 (2006).

${ }^{10}$ L. Gui, B. Xu, and T. C. Chong, IEEE Photon. Technol. Lett. 16, 1337 (2004).

${ }^{11}$ J. Burghoff, S. Nolte, and A. Tuennermann, Appl. Phys. A: Mater. Sci. Process. 89, 127 (2007).

${ }^{12}$ M. Arbore M. Fejer, M. Fermann, A. Hariharan, A. Galvanauskas, and D. Harter, Opt. Lett. 22, 13 (1997).

${ }^{13}$ M. Dunn and M. Ebrahimzadeh, Science 286, 1513 (1999).

${ }^{14}$ C. Schmuttenmaer, Chem. Rev. 104, 1759 (2004).

${ }^{15}$ Y. Lee, T. Meade, V. Perlin, H. Winful, T. Norris, and A. Galvanauskas, Appl. Phys. Lett. 76, 2505 (2000).

${ }^{16}$ E. G. Gamaly, S. Juodkazis, V. Mizeikis, H. Misawa, A. V. Rode,
W. Krolikowski, and K. Kitamura, Curr. Appl. Phys. 8, 416 (2008).

${ }^{17}$ S. Juodkazis, V. Mizeikis, M. Sudžius, H. Misawa, K. Kitamura, S. Takekawa, E. G. Gamaly, W. Krolikowski, and A. V. Rode, Appl. Phys. A: Mater. Sci. Process. 93, 129 (2008).

${ }^{18}$ D. Arnold and E. Cartier, Phys. Rev. B 46, 15102 (1992).

${ }^{19}$ S. Juodkazis, A. V. Rode, E. G. Gamaly, S. Matsuo, and H. Misawa, Appl. Phys. B: Lasers Opt. 77, 361 (2003).

${ }^{20}$ G. C. Valley, IEEE J. Quantum Electron. 19, 1637 (1983).

${ }^{21}$ Inorganic crystal structure database (icsd) structure code: 155360. Accessible at http://icsdweb.fiz-karlsruhe.de/index.html

${ }^{22}$ C. Mauclair, A. Mermillod-Blondin, N. Huot, E. Audouard, and R. Stoian, Opt. Express 16, 5481 (2008).

${ }^{23}$ K. Buse, Appl. Phys. B: Lasers Opt. 64, 273 (1997).

${ }^{24}$ G. Panotopoulos, M. Luennemann, K. Buse, and D. Psaltis, J. Appl. Phys. 92, 793 (2002).

${ }^{25}$ B. I. Sturman and V. M. Fridkin, The Photovoltaic and Photorefractive Effects in Non Centrosymmetric Materials, Ferroelectricity and Related Phenomena (Gordon and Breach, New York, 1992), Vol. 8.

${ }^{26}$ F. Jermann and J. Otten, J. Opt. Soc. Am. B 10, 2085 (1993).

${ }^{27}$ K. B. M. Simon, St. Wevering, and E. Krätzig, J. Phys. D 30, 141 (1997).

${ }^{28}$ D. N. Nikogosyan, Properties of Optical and Laser-Related Materials: A Handbook (Wiley, New York, 1988).

${ }^{29}$ Y. Il'insky and L. Keldysh, Electromagnetic Response of Material Media (Plenum, New York, 1988).

${ }^{30}$ A. M. Glass, D. von der Linde, and T. J. Negran, Appl. Phys. Lett. 25, 233 (1974). 
${ }^{31}$ Y. Raizer, Gas Discharge Physics (Springer, Berlin, 1991).

${ }^{32}$ E. G. Gamaly, A. V. Rode, and B. Luther-Davies, Appl. Phys. A: Mater. Sci. Process. 69, S121 (1999).

${ }^{33}$ C. Kittel, Introduction to Solid State Physics, 7th ed. (Wiley, New York, 1996).

${ }^{34}$ Y. P. Raizer, Laser-Induced Discharge Phenomena (Consultant Bureau, New York, 1977).

${ }^{35}$ E. G. Gamaly, A. Rode, B. Luther-Davies, and V. Tikhonchuk, Phys. Plasmas 9, 949 (2002).

${ }^{36}$ G. Ghosh, Opt. Lett. 19, 1391 (1994).

${ }^{37}$ M. Petrov, S. I. Stepanov, and A. Khomenko, in Photorefractive
Crystals in Coherent Optical Systems (Springer-Verlag, Berlin, 1991).

${ }^{38}$ A. Marcinkevičius, V. Mizeikis, S. Juodkazis, S. Matsuo, and H. Misawa, Appl. Phys. A: Mater. Sci. Process. 76, 257 (2003).

${ }^{39}$ O. Beyer, I. Breuning, F. Kalkum, and K. Buse, Appl. Phys. Lett. 88, 051120 (2006).

${ }^{40}$ O. Beyer, D. Maxein, K. Buse, B. I. Sturman, H. Hsieh, and D. Psaltis, Opt. Lett. 30, 1366 (2005).

${ }^{41}$ O. Beyer, D. Maxein, K. Buse, B. Sturman, H. T. Hsieh, and D. Psaltis, Phys. Rev. E 71, 056603 (2005). 